\title{
PORTAL DOS BEBÊS - SEÇÃO APARELHO AUDITIVO: AVALIAÇÃO POR PAIS DE CRIANÇAS DEFICIENTES AUDITIVAS
}

\author{
Babies' portal website - hearing aid section: \\ hearing impaired children parents' assessment
}

Bárbara Guimarães Bastos (1), Deborah Viviane Ferrari (2)

\section{RESUMO}

Objetivo: verificar a auto-avaliação do conhecimento dos pais de crianças deficientes auditivas sobre o Aparelho de Amplificação Sonora Individual (AASI) e molde auricular e como o conteúdo do website "Portal dos Bebês - Seção Aparelhos Auditivos" auxiliou no entendimento de tais informações. Métodos: 22 pais (seis homens e 16 mulheres, idade média 32,6 anos) preencheram anonimamente um formulário online com 36 questões abrangendo dados demográficos, uso da internet, orientação fornecida no momento da adaptação do AASI e auxílio fornecido pelo conteúdo do website. Resultados: no dia da adaptação do AASI materiais informativos foram oferecidos apenas para $52,4 \%$ dos pais, no entanto, estes estavam satisfeitos com as orientações recebidas. Em média o auxílio fornecido pelo conteúdo do website foi de $86 \%$. A pontuação atribuída ao conteúdo e a satisfação com o website foi relacionada à idade da criança, tempo de uso do AASI e conhecimento prévio dos pais a respeito dos tópicos sobre o AASI e molde. Conclusões: o "Portal dos Bebês" é um instrumento que pode auxiliar pais de crianças deficientes auditivas na compreensão das orientações fornecidas no momento da adaptação do AASI. A continuidade do estudo com maior número de participantes é necessária.

DESCRITORES: Perda Auditiva; Auxiliares de Audição; Audiologia; Aconselhamento; Telemedicina; Internet

\section{INTRODUÇÃO}

A responsabilidade pelas decisões sobre 0 uso e cuidados durante a rotina diária e resolução de problemas no funcionamento do aparelho de amplificação sonora individual (AASI) em bebês e crianças é, em última instância, da família. Assim, estes indivíduos devem ser corretamente orientados de modo a assegurar a utilização apropriada dos AASIs.

(1) Programa de Fonoaudiologia da Faculdade de Odontologia de Bauru - Universidade de São Paulo - FOB/USP, Bauru, SP, Brasil.

(2) Departamento de Fonoaudiologia da Faculdade de Odontologia de Bauru da Universidade de São Paulo - FOB/ USP, Bauru, SP, Brasil.

Trabalho realizado no Departamento de Fonoaudiologia da Faculdade de Odontologia de Bauru da Universidade de São Paulo.

Fonte de Auxílio: FAPESP (processo número: 2008/07963-0)

Conflito de interesses: inexistente
Os profissionais frequentemente relatam que os pais não se lembram ou não compreendem adequadamente as orientações oferecidas e, por consequência, não asseguram a utilização efetiva do AASI pela criança, não realizam os cuidados diários ao AASI e não tomam providências em casos de mau-funcionamento ${ }^{1}$.

A dificuldade de retenção ou não entendimento das informações é resultado, dentre outros fatores, do discurso técnico empregado por muitos profissionais ${ }^{2}$ e das reações emocionais dos pais frente ao diagnóstico da deficiência auditiva e adaptação do AASI ${ }^{3}$. Durante o período que compreende o diagnóstico audiológico e o início da adaptação do AASI os pais precisam de tempo para absorver as informações e reagir emocionalmente a elas. Se muita informação técnica for oferecida neste momento, grande parte dos pais pode ser incapaz de processá-la ${ }^{2}$.

Para que os pais/responsáveis compreendam as orientações oferecidas é necessário que estas 
sejam claras, acessíveis e realizadas de maneira sistemática ${ }^{4,5}$. As informações verbais devem ser complementadas com materiais educativos com linguagem simples e acessível, para que os pais acessem após as consultas, respeitando assim o tempo para assimilação do conteúdo ${ }^{6}$.

O "Portal dos Bebês - seção aparelho auditivo" foi criado para pais e cuidadores de crianças deficientes auditivas, com idade entre zero a três anos, a fim de permitir o acesso conveniente e diário às orientações quanto ao uso e cuidados com o AASI e molde auricular, bem como a resolução dos principais problemas encontrados durante a utilização dos mesmos.

O objetivo deste trabalho foi verificar a auto-avaliação do conhecimento dos pais de crianças deficientes auditivas sobre 0 Aparelho de Amplificação Sonora Individual (AASI) e molde auricular e como o conteúdo do website "Portal dos Bebês - Seção Aparelhos Auditivos" auxiliou no entendimento de tais informações.

\section{MÉTODOS}

Estudo descritivo transversal, realizado no Departamento de Fonoaudiologia da Faculdade de Odontologia de Bauru, Universidade de São Paulo.

Recebeu análise e aprovação do Comitê de Ética em Pesquisa da FOB-USP (processo número 009/2009).

De acordo com a Portaria SAS $587^{7}$ crianças de até três anos de idade são atendidas em serviços de saúde auditiva de alta complexidade. Um levantamento realizado em maio de 2010 indicou a existência de 79 serviços desta natureza credenciados no Sistema Único de Saúde ${ }^{8}$. Tais serviços foram contatados a fim de informar o propósito do estudo e obter autorização para envio do convite de participação, via correio. Foi solicitado aos responsáveis técnicos e profissionais destes serviços que distribuíssem os convites de participação aos pais de crianças deficientes auditivas.

Além disto, também foi realizada a divulgação do website e convite de participação na pesquisa no fórum de implante coclear (FIC), hospedado no website Yahoo e em redes sociais (Facebook e
Orkut) os quais apresentam comunidades para pais de crianças deficientes auditivas. A Associação dos Deficientes Auditivos, Pais, Amigos e Usuários de Implante Coclear de Bauru (ADAP) também enviou o folder de divulgação do website "Portal dos Bebês" para os e-mails de seus associados.

O convite continha uma explicação sobre o propósito do estudo e o endereço para acesso e navegação no website "Portal dos Bebês" bem como o link "Avalie este site". Neste link constava - Termo de Consentimento Livre e Esclarecido (TCLE) e o formulário de avaliação online.

No período de coleta de dados do presente estudo, 39 pais acessaram o link "Avalie este site" do "Portal dos Bebês". Destes, 17 indivíduos foram excluídos, pois não preencheram o formulário de avaliação do site. Assim, participaram voluntariamente do estudo, após assinatura do TCLE, 22 indivíduos (seis homens e 16 mulheres) com idades entre 17 e 62 anos (média 32,6 anos) pais e mães de crianças com deficiência auditiva neurossensorial unilateral $(n=1 ; 4,5 \%)$ e bilateral $(n=21,95,5 \%)$ de grau leve $(n=1 ; 4,5 \%)$, moderado $(n=5 ; 22,7 \%)$, severo $(n=1 ; 4,5 \%)$ e profundo $(n=15 ; 68,2 \%)$.

No que se refere à escolaridade, os participantes possuíam ensino fundamental completo $(n=3 ; 13,6 \%)$ e incompleto $(n=1 ; 4,6 \%)$, ensino médio completo $(n=5 ; 22,7 \%)$, ensino superior completo $(n=5 ; 22,7 \%)$ e incompleto $(n=7 ; 31,8 \%)$ e mestrado $(n=1,4,6 \%)$.

Quanto à região de residência os participantes estavam divididos em: Sudeste $(n=15 ; 68,2 \%)$, Sul $(n=3 ; 13,6 \%)$, Norte $(n=2 ; 9,1 \%)$, Nordeste $(n=1 ; 4,5 \%)$ e Centro-Oeste $(n=1 ; 4,5 \%)$. Para fins da análise de dados os participantes das regiões Norte, Nordeste e Centro-Oeste foram agrupados, formando a região NNC ( $n=4 ; 18,2 \%)$. Doze participantes $(54,5 \%)$ realizavam o tratamento da criança na cidade de Bauru/São Paulo, seis $(27,3 \%)$ em Sorocaba/São Paulo e quatro (18,2\%) em outras cidades (Campinas, Alfenas, São Mateus e São Paulo).

$\mathrm{Na}$ Tabela 1 encontram-se os dados da idade da criança no momento da coleta de dados, a idade na época do diagnóstico audiológico e na adaptação dos AASIs. 
Tabela 1 - Idade da criança no momento da coleta de dados (atual), na época do diagnóstico audiológico e na adaptação dos AASIs e tempo de adaptação dos dispositivos, por região do país $(n=22)$

\begin{tabular}{|c|c|c|c|c|c|}
\hline \multirow{2}{*}{\multicolumn{2}{|c|}{ Dados da Criança }} & \multicolumn{3}{|c|}{ Região } & \multirow{2}{*}{$\begin{array}{c}\text { Total } \\
(\mathrm{n}=22)\end{array}$} \\
\hline & & $\begin{array}{c}\text { Sudeste } \\
(n=15)\end{array}$ & $\begin{array}{c}\text { Sul } \\
(n=3)\end{array}$ & $\begin{array}{l}\text { NNC } \\
(n=4)\end{array}$ & \\
\hline \multicolumn{6}{|l|}{ Idade (meses) } \\
\hline Atual & $\bar{x} \pm d p$ & $60,1 \pm 45,9$ & $21,0 \pm 7,8$ & $50,7 \pm 49,3$ & $53,0 \pm 44,1$ \\
\hline Diagnóstico & $\bar{x} \pm d p$ & $33,4 \pm 31,5$ & $15,0 \pm 12,12$ & $12,0 \pm 9,2$ & $27,0 \pm 27,9$ \\
\hline \multirow{2}{*}{$\begin{array}{c}\text { Adaptação } \\
\text { Tempo adaptação } \\
\text { (meses) }\end{array}$} & $\bar{x} \pm d p$ & $30,9 \pm 25,6$ & $19,0 \pm 11,2$ & $15,5 \pm 9,9$ & $26,5 \pm 22,6$ \\
\hline & $\bar{x} \pm d p$ & $29,2 \pm 35,5$ & $2,0 \pm 3,4$ & $35,2 \pm 40,8$ & $26,5 \pm 34,4$ \\
\hline
\end{tabular}

Legenda: NNC: Norte, Nordeste e Centro-Oeste; Média: $\bar{x}$; Desvio Padrão: dp

Os participantes foram solicitados a acessar e navegar pelo website "Portal dos Bebês" (http:// portaldosbebes.fob.usp.br) e, posteriormente, preencher anonimamente um formulário de avaliação online, composto por cinco partes.

\section{$1^{\text {a }}$ parte}

Continha informações sobre o questionário e o termo de consentimento livre e esclarecido, com duas opções de resposta: "Eu não quero participar" (o usuário era direcionado a uma página de agradecimento, não tendo acesso ao formulário de avaliação) e "Eu quero participar" (o indivíduo era direcionado aos questionários de avaliação).

\section{$2^{\mathrm{a}}$ parte (questões 2 a 18)}

Consistia de questões relacionadas aos dados demográficos dos participantes, o uso da internet e informações sobre a idade e perda de audição da criança. Seis questões eram abertas, quatro eram semi fechadas e duas eram fechadas.

\section{$3^{\text {a }}$ parte (questões 19 a 22)}

Compreendia questões a respeito do aconselhamento informativo oferecido no dia em que o AASI foi adaptado na criança. A questão 22 era aberta e permitia a identificação dos materiais recebidos no momento da adaptação do AASI, caso isto tivesse ocorrido.

Exemplo: "Considerando o dia em que a criança recebeu o aparelho auditivo, como você julgaria sua satisfação com a quantidade de tempo usado pelo profissional para Ihe passar as orientações de uso e cuidado com os aparelhos?
( ) muito insatisfeito ( ) insatisfeito
( ) nem insatisfeito e nem satisfeito (neutro)
( ) satisfeito ( ) muito satisfeito"

\section{$4^{\mathrm{a}}$ parte (questões 23 a 30 )}

Continha questões a respeito do conteúdo do "Portal dos Bebês". Inicialmente (questão 23) os pais realizaram a auto-avaliação do seu conhecimento sobre os aparelhos de amplificação sonora individuais, antes de acessar o "Portal dos Bebês", por meio de uma escala Likert de cinco pontos que variava de "muito ruim" a "muito bom".

"Antes de visitar o website "Portal dos Bebês

- Seção Aparelhos Auditivos", como você avaliaria o seu conhecimento geral:

( ) muito ruim ( ) ruim ( ) satisfatório ( ) bom ( ) muito bom"

As questões 24 a 30 consistiam da avaliação dos pais ou cuidadores a respeito de como o website ajudou, em relação ao seu conhecimento prévio, a melhorar o entendimento de um determinado conteúdo sobre o AASI. Os conteúdos abordados foram: conhecimento geral a respeito dos AASIs, funcionamento do AASI, para que serve o AASI, diferentes tipos e tecnologias, dúvidas em relação à limpeza dos moldes auriculares e AASIs, verificação e resolução de problemas e como criar uma rotina de uso diário dos AASIs.

Exemplo: "Tendo como comparação o seu conhecimento prévio, o quanto o website lhe ajudou a entender para que serve um apareIho auditivo?
( ) não ajudou ( ) ajudou pouco
( ) ajudou ( ) ajudou muito
( ) não acessel"

Nas questões 24 a 30 pontos eram atribuídos para cada opção de resposta, variando de um ponto ("não ajudou") a quatro pontos ("ajudou muito"). A opção "não acessei esta parte" não era pontuada 
ou incluída na análise. Para esta parte do formulário foi realizado o cálculo do total de pontos atribuídos na avaliação feita pelos pais em comparação à pontuação possível de ser obtida. A pontuação possível era igual ao número de questões respondidas multiplicado por quatro pontos. Sendo assim, a pontuação total possível também era particular a um dado preenchimento do questionário.

A pontuação final era dada pelo total de pontos obtidos dividido pela pontuação total possível. A fim de obter uma porcentagem este resultado foi multiplicado por 100.

$\frac{\text { Pontuação total obtida }}{\text { Pontuac̃ão }} \times 100=$ Porcentagem final Pontuação total possível

Por exemplo: $\underline{24}$ x $100=85.7 \%$ 28

\section{5- parte (questões 31 a 35)}

As questões 31 e 32 eram de múltipla escolha sendo o participante solicitado a escolher uma resposta dentro cinco alternativas. A questão 31 versava sobre o quanto as dicas fornecidas no website aumentariam o uso do AASI pela criança e as alternativas variavam de "nem um pouco" (um ponto) a "muitíssimo" (cinco pontos). A questão 32 dizia respeito à satisfação geral com o website e as opções de resposta variavam de "muito insatisfeito" (um ponto) a "muito satisfeito" (cinco pontos).

A questão 33 era aberta e solicitava aos pais que elencassem as três principais dificuldades que encontravam com os AASIs de seus filhos antes do acesso ao website. A questão 34 (múltipla escolha) solicitava aos participantes que escolhessem, dentre quatro alternativas que variaram de "nem um pouco" (um ponto) a "ajudou muito" (quatro pontos), o quanto o website ajudou a esclarecer / sanar as dificuldades elencadas na pergunta 33.
Na questão 35 foi perguntado aos participantes se estes indicariam o "Portal dos Bebês" para outros pais de crianças deficientes auditivas, sendo possível selecionar "sim" ou "não".

Ao final do formulário foi disponibilizado um espaço para o participante fazer comentários ou sugestões que julgassem pertinentes.

Este trabalho foi analisado e aprovado pelo Comitê de Ética em Pesquisa da instituição de origem (processo número 009/2009).

As respostas quanto à satisfação em relação às orientações recebidas no momento da adaptação foram analisadas por meio de estatística descritiva.

O teste não paramétrico de Mann-Whitney foi utilizado para verificar a diferença entre a autoavaliação do conhecimento prévio a respeito do AASI entre aqueles que receberam materiais no momento do aconselhamento e aqueles que não receberam.

Foi verificada a correlação (Spearman) entre a avaliação do conteúdo e satisfação com o website com o conhecimento prévio sobre o AASI.

$O$ teste de Friedman foi utilizado para analisar as diferenças nas avaliações dos participantes sobre como o website os ajudou a melhorar o entendimento de um determinado conteúdo a respeito do AASI e/ou molde auricular.

Em todos os casos, o nível de significância adotado foi igual a $5 \%$.

\section{RESULTADOS}

O tempo de preenchimento do formulário variou de 12 minutos a 1 hora e 56 minutos (média de 47,1 minutos; $d p \pm 35,5$ ).

Nas Tabelas 2 e 3 encontram-se os dados referentes à frequência e local de acesso à internet e velocidade de internet utilizada. 
Tabela 2 - Frequência de acesso à internet pelos pais e cuidadores, por região do país ( $\mathrm{n}=\mathbf{2 2})$

\begin{tabular}{|c|c|c|c|c|c|c|}
\hline \multirow{3}{*}{ Regiões } & \multicolumn{3}{|c|}{ Frequência uso internet } & \multicolumn{3}{|c|}{ Local uso internet } \\
\hline & $\begin{array}{c}\text { Frequentemente } \\
\text { (Várias vezes ao } \\
\text { dia) }\end{array}$ & $\begin{array}{c}\text { Geralmente e } \\
\text { Ocasionalmente } \\
\text { (várias vezes, ou } \\
\text { uma ou duas } \\
\text { vezes na } \\
\text { semana) }\end{array}$ & $\begin{array}{c}\text { Quase nunca } \\
\text { (menos que uma } \\
\text { vez ao mês) }\end{array}$ & Casa & Trabalho & Outro \\
\hline & $n(\%)$ & $\mathrm{n}(\%)$ & $n(\%)$ & $n(\%)$ & $n(\%)$ & $n(\%)$ \\
\hline $\begin{array}{c}\text { Sudeste } \\
(n=15)\end{array}$ & $6(40 \%)$ & $3(20 \%)$ & $6(40 \%)$ & $7(46,6 \%)$ & $4(26,7 \%)$ & $4(26,7 \%)$ \\
\hline $\begin{array}{c}\text { Sul } \\
(n=3)\end{array}$ & $1(33,3 \%)$ & $1(33,3 \%)$ & $1(33,3 \%)$ & $1(33,3 \%)$ & 0 & $2(66,4 \%)$ \\
\hline $\begin{array}{l}\text { NNC } \\
(n=4)\end{array}$ & $2(50 \%)$ & $1(25 \%)$ & $1(25 \%)$ & $4(100 \%)$ & 0 & 0 \\
\hline $\begin{array}{c}\text { Total } \\
(n=22)\end{array}$ & $9(40,9 \%)$ & $5(22,7 \%)$ & $8(36,4 \%)$ & $12(54,5 \%)$ & $4(18,2 \%)$ & $6(27,3 \%)$ \\
\hline
\end{tabular}

Legenda: NNC: Norte, Nordeste e Centro-Oeste

Tabela 3 - Velocidade da internet utilizada pelos pais e cuidadores, por região do país $(n=22)$

\begin{tabular}{ccccccccc}
\hline & \multicolumn{7}{c}{ Velocidade da internet } \\
\cline { 2 - 9 } Regiões & \multicolumn{2}{c}{ Banda larga } & \multicolumn{2}{c}{ Internet discada } & Não soube informar & \multicolumn{2}{c}{ Não acessa } \\
\cline { 2 - 9 } & $\mathbf{n}$ & $\%$ & $\mathbf{n}$ & $\%$ & $\mathbf{n}$ & $\%$ & $\mathbf{n}$ & $\%$ \\
\hline $\begin{array}{c}\text { Sudeste } \\
(\mathbf{n}=\mathbf{1 5}) \\
\text { Sul }\end{array}$ & 9 & 60 & 1 & 6,7 & 4 & 26,6 & 1 & 6,7 \\
$\begin{array}{c}(\mathbf{n}=\mathbf{3}) \\
\mathbf{N N C}\end{array}$ & 2 & 66,7 & 0 & 0 & 1 & 33,3 & 0 & 0 \\
$(\mathbf{n}=\mathbf{4})$ & 1 & 25 & 1 & 25 & 2 & 50 & 0 & 0 \\
$\begin{array}{c}\text { Total } \\
(\mathbf{n}=\mathbf{2 2})\end{array}$ & 12 & 54,5 & 2 & 9,1 & 7 & 31,8 & 1 & 4,5 \\
\hline
\end{tabular}

Legenda: NNC: Norte, Nordeste e Centro-Oeste

Ressalta-se que um participante não respondeu as perguntas 24 a 36 do formulário, desta forma algumas análises mostraram um total de 21 participantes. A Tabela 4 mostra a percepção dos pais com relação ao tempo disponibilizado para orientação e clareza das informações fornecidas pelo profissional no dia da adaptação dos AASIs em seus filhos. Todos os participantes relataram se sentir a vontade para fazer perguntas ao profissional no dia da adaptação do AASI. 
Tabela 4 - Avaliação da satisfação dos participantes (0 a 5$)$ com relação ao tempo disponibilizado para orientação e clareza das informações oferecidas no momento da adaptação do AASI, de acordo com a região $(n=21)$

\begin{tabular}{|c|c|c|c|c|c|c|c|c|c|c|}
\hline \multirow{3}{*}{ Região } & \multicolumn{10}{|c|}{ Satisfação Atendimento } \\
\hline & \multicolumn{5}{|c|}{ Tempo disponível } & \multicolumn{5}{|c|}{ Clareza das informações } \\
\hline & $\overline{\mathbf{x}}$ & $d p$ & mín & máx & md & $\overline{\mathbf{x}}$ & $d p$ & mín & máx & md \\
\hline $\begin{array}{c}\text { Sudeste } \\
(n=14)\end{array}$ & 4,1 & 1,26 & 1 & 5 & 4,5 & 4,1 & 1,26 & 1 & 5 & 4,5 \\
\hline $\begin{array}{c}\text { Sul } \\
(n=3)\end{array}$ & 4,3 & 0,57 & 4 & 5 & 4 & 3,3 & 2,08 & 1 & 5 & 4 \\
\hline $\begin{array}{l}\text { NNC } \\
(n=4)\end{array}$ & 4,5 & 0,57 & 4 & 5 & 4,5 & 4,5 & 0,57 & 4 & 5 & 4,5 \\
\hline $\begin{array}{l}\text { Total } \\
(n=21)\end{array}$ & 4,1 & 1,07 & 1 & 5 & 4 & 4,0 & 1,28 & 1 & 5 & 4 \\
\hline
\end{tabular}

Legenda: NNC: Norte, Nordeste e Centro-Oeste; Média: $\bar{x}$;Desvio Padrão: dp; Mínimo: mín; Máximo: máx; Mediana: md

Apenas onze participantes $(52,4 \%)$ receberam materiais informativos no dia da adaptação do AASI, sendo estes manuais dos fabricantes de AASI e livretos com informações sobre uso e cuidados com este dispositivo. A Figura 1 mostra a auto-avaliação dos pais a respeito do conhecimento prévio sobre os tópicos relacionados ao aparelho de amplificação sonora individual, considerando o recebimento ou não de material informativo (questão 23).

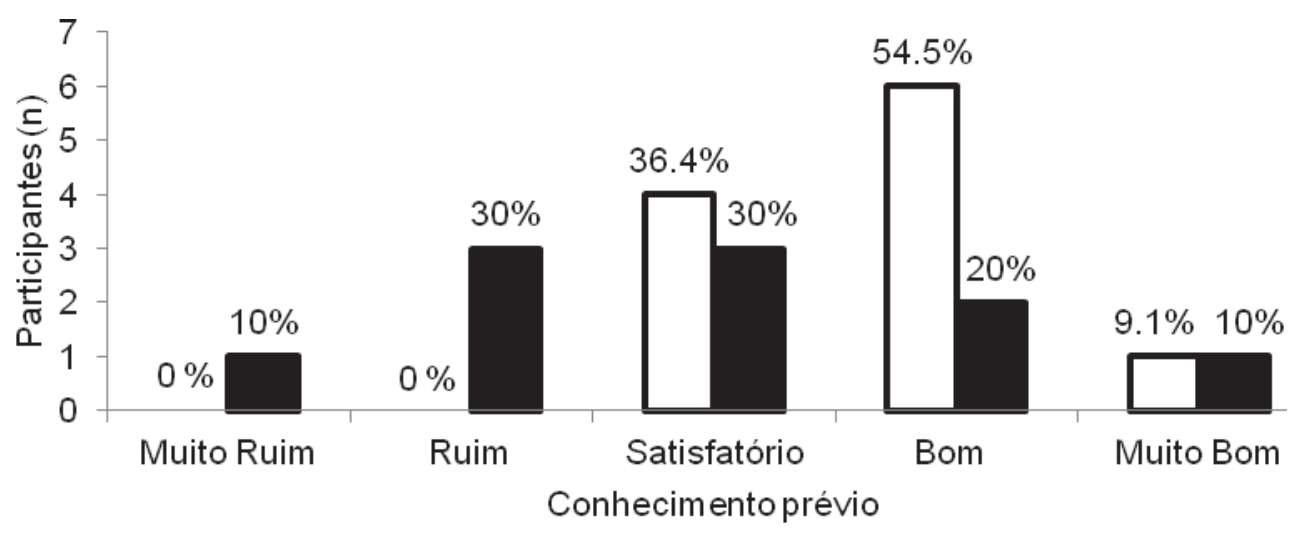

QReceberam material informativo

Não receberam material informativo

Figura 1 - Distribuição dos participantes que receberam e não receberam material informativo, quanto à auto-avaliação do conhecimento prévio sobre o aparelho de amplificação sonora individual $(\mathrm{n}=21)$

O teste de Mann-Whitney revelou diferenças estatisticamente significantes entre os grupos $\left(z=2,60\right.$ e $\left.p=0,00^{*}\right)$ que receberam e não receberam materiais informativos no dia da adaptação em relação à auto-avaliação do conhecimento prévio a respeito sobre o AASI.
A Tabela 5 mostra a pontuação dada pelos participantes sobre como o website auxiliou na melhora do entendimento de diferentes tópicos sobre o aparelho de amplificação sonora individual. Um máximo de quatro pontos poderia ser obtido em cada item. 
Tabela 5 - Análise descritiva da pontuação sobre como o website auxiliou na melhora do entendimento de diferentes tópicos sobre o aparelho de amplificação sonora individual

\begin{tabular}{|c|c|c|c|c|}
\hline \multirow[t]{2}{*}{ Item } & \multicolumn{2}{|c|}{ Todos os participantes } & \multicolumn{2}{|c|}{$\begin{array}{l}\text { Grupo para análise } \\
\text { estatística }(n=15)\end{array}$} \\
\hline & $\overline{\mathbf{x}}$ & dp & $\bar{x}$ & dp \\
\hline Funcionamento do AASI $(n=20)$ & 3,4 & 0,59 & 3,5 & 0,51 \\
\hline Para que serve um AASI $(n=19)$ & 3,3 & 0,68 & 3,4 & 0,51 \\
\hline Tecnologia dos AASI $(n=19)$ & 3,5 & 0,61 & 3,5 & 0,63 \\
\hline Tipos de AASI $(n=18)$ & 3,5 & 0,51 & 3,5 & 0,51 \\
\hline Limpeza do AASI e molde auricular $(n=19)$ & 3,2 & 0,85 & 3,4 & 0,63 \\
\hline $\begin{array}{l}\text { Verificação do AASI/Resolução de } \\
\text { problemas }(n=20)\end{array}$ & 3,1 & 0,58 & 3,2 & 0,59 \\
\hline $\begin{array}{l}\text { Como criar uma rotina de uso do aparelho } \\
\text { auditivo }(n=18)\end{array}$ & 3,4 & 0,70 & 3,6 & 0,50 \\
\hline Total $\%(n=21)$ & 84,0 & 12,11 & 86,9 & 10,42 \\
\hline
\end{tabular}

Média $(\bar{x})$ e Desvio Padrão (dp)

Para a análise estatística com o teste de Friedman foram considerados apenas os 15 participantes que acessaram e avaliaram todos os sete conteúdos indicados no formulário de avaliação. As médias das pontuações dos itens foram comparadas, não sendo encontradas diferenças significantes entre eles $(x 2=6,70$ e $p=0,34)$.

No que se refere à satisfação com o website, os pais relataram estar muito satisfeitos $(n=11$, $52,4 \%)$; satisfeitos $(n=5,23,8 \%)$, neutros $(n=1$, $4,8 \%)$, insatisfeitos ( $n=1,4,8 \%$ ) e muito insatisfeitos $(n=3,14,2 \%)$.
Houve correlação (Spearman) negativa significante $\left(r h o=-0,55\right.$ e $\left.p=0,01^{*}\right)$ entre conhecimento prévio a respeito dos AASIs com a pontuação total dada ao conteúdo do website. Não foi encontrada correlação entre o conhecimento prévio a respeito dos tópicos em AASI e satisfação com o website (rho $=-0,14$ e $p=0,52$ ).

A Tabela 6 mostra as principais dificuldades elencadas pelos pais a respeito do uso do aparelho de amplificação sonora individual na criança.

Tabela 6 - Principais dificuldades elencadas pelos pais a respeito do uso do aparelho de amplificação sonora individual na criança $(n=21)$

\begin{tabular}{cc}
\hline Dificuldades & $\%$ \\
\hline Habituação da criança com o uso do AASI & 21,4 \\
Uso e cuidados com o AASI & 14,3 \\
Limitação do uso do AASI em algumas atividades (ex: nadar) & 14,3 \\
Fixação do AASI na orelha & 14.3 \\
Tempo de uso diário do AASI & 14.3 \\
Estigma do AASI & 10.7 \\
Confecção dos moldes auriculares & 7.1 \\
Aceitação da perda auditiva & 3.6 \\
\hline
\end{tabular}

A Figura 2 mostra a opinião dos pais do quanto o website os ajudou a sanar as dificuldades elencadas na Tabela 6.

Onze participantes $(50 \%)$ enviaram comentários, dos quais quatro foram excluídos da análise por tratarem de assuntos alheios à avaliação do website. Os demais sete comentários foram agrupados por categorias (Figura 3).

Dos 19 respondentes da questão sobre a recomendação do acesso ao website, 100\% relataram que indicariam o website para outros pais de crianças deficientes auditivas usuárias de AASI. 


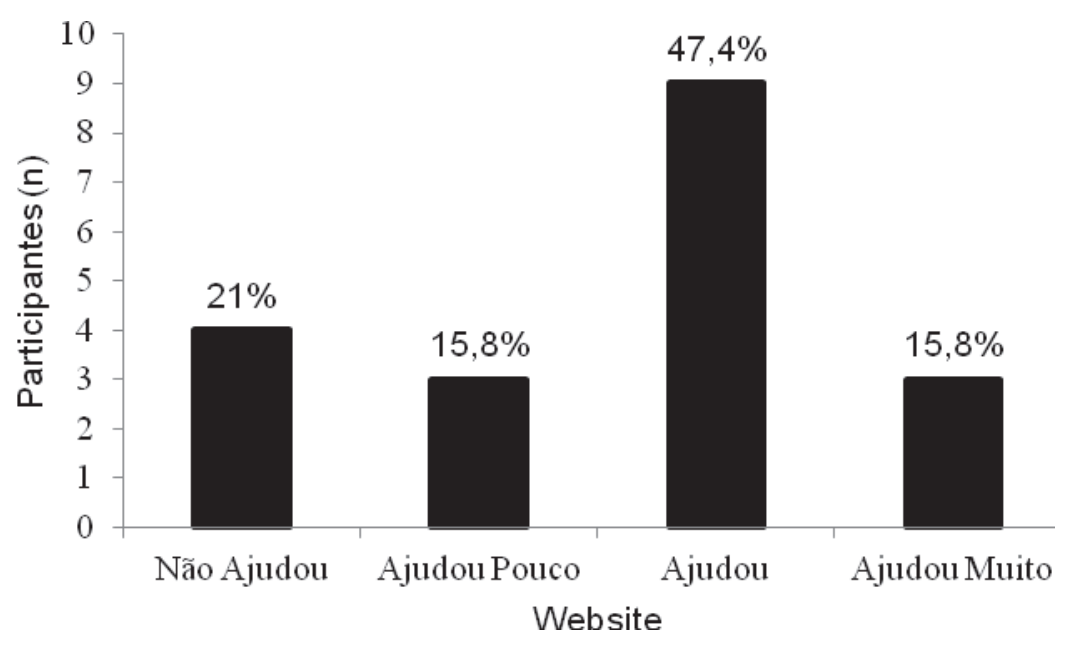

Figura 2 - Opinião dos pais de quanto o website os ajudou a sanar as dificuldades a respeito do uso do aparelho de amplificação sonora individual na criança

\begin{tabular}{|c|l|}
\hline \multirow{5}{*}{ Conteúdo } & $\begin{array}{l}\text { "Gostaria de receber informações sobre os avanços tecnológicos e também } \\
\text { sobre formas de terapias relacionados à idade e ao nível de compreensão do } \\
\text { meu filho" } \\
\text { "Acrescentar dicas para as crianças na fase de adolescência de como lidar } \\
\text { com perguntas (...)." }\end{array}$ \\
\hline \multirow{2}{*}{ Estrutura } & $\begin{array}{l}\text { "O link para pais e cuidadores avaliarem o portal está desconfigurado, } \\
\text { aparecendo apenas o pa bem no cantinho." } \\
\text { "Necessidade de mais informações com relação ao tipo de tratamento para os } \\
\text { médicos." } \\
\text { "No momento o site não contribuiu muito porque já tenho uma grande } \\
\text { experiência na rotina com os aparelhos, a criança usa há } 6 \text { anos. Acredito que } \\
\text { o site contribui para quem está começando este processo, é uma ferramenta } \\
\text { para auxiliar a resolver os problemas que surgem." } \\
\text { "Que esse site tenha uma maior divulgação para ajudar as mães que } \\
\text { acabaram de descobrir que o filho não escuta, porque no começo do } \\
\text { tratamento tudo é mais difícil." }\end{array}$ \\
\hline Relevância & $\begin{array}{l}\text { "Só tenho a agradecer todas as informações do site que serviram de grande } \\
\text { ajuda tanto pra mim como para o meu filho." }\end{array}$ \\
\hline
\end{tabular}

Figura 3 - Comentários dos pais

\section{DISCUSSÃO}

Primeiramente faz-se necessário esclarecer que, considerando o universo de crianças atendidas, sobretudo no Sistema Único de Saúde, era esperado que aproximadamente 100 pais e/ou cuidadores participassem da pesquisa. No entanto, a despeito dos esforços de divulgação verificou-se uma baixa adesão desta população o que limita a discussão dos resultados do presente estudo.

A literatura ${ }^{9}$ relata taxas de adesão de $25 \%$ de respondentes de pesquisas com questionários aplicados online e via e-mail. Outro fator que afeta a aderência em pesquisas online é o tamanho do questionário a ser respondido, sendo que questionários muito longos levam ao desinteresse por parte do potencial participante ${ }^{10}$. O formulário utilizado para os pais continha 36 questões e isto também pode ter contribuído para a baixa aderência dos pais na participação na pesquisa.

Alguns profissionais que trabalhavam nos serviços de saúde auditiva de alta complexidade entraram em contato com a pesquisadora informando que embora os pais/cuidadores tivessem acessado o website os mesmos não responderam o questionário em função da indisponibilidade de 
tempo e pelo acesso limitado à internet. Em outros casos, os profissionais relataram que o próprio curso de tratamento da criança (por exemplo, crianças que estavam em avaliação para indicação do implante coclear) impactava o estado emocional dos pais que, por isto, não se sentiram motivados a participar da pesquisa.

Os participantes se sentiram a vontade para fazer perguntas ao profissional no momento da adaptação do AASI em seus filhos e estavam satisfeitos com o tempo disponível e clareza das informações fornecidas (Tabela 4). No que se refere à clareza das informações, os participantes da região Sul estavam menos satisfeitos do que os das outras regiões, no entanto esta análise torna-se limitada em função do número de pacientes neste grupo. Também em virtude do número de participantes não foi possível aplicar estatística indutiva nestes dados. Feita a ressalva em relação ao tamanho da amostra estudada, os resultados do presente estudo foram mais favoráveis do que os relatados na literatura ${ }^{4,5,11-13}$.

Apenas onze participantes $(52,4 \%)$ receberam material informativo no momento da adaptação. Embora a qualidade de estrutura, design, layout e abrangência de conteúdos possam variar, todos os fabricantes de AASI fornecem um manual que acompanha o dispositivo. Desta forma, muito provavelmente a indisponibilidade de material não é o fator que levou a estes resultados. Este dado é preocupante tendo em vista os baixos índices de retenção de informação reportados para adultos ${ }^{14}$ e para pais de crianças deficientes auditivas ${ }^{15}$. Pais e cuidadores necessitam do fornecimento de materiais escritos para analisar posteriormente 5,12 tendo acesso à informação de forma mais conveniente e quantas vezes necessitarem ${ }^{11}$.

Os pais que receberam materiais informativos no dia da adaptação do AASI auto avaliaram seu conhecimento a respeito destes dispositivos, previamente ao acesso ao "Portal dos Bebês", de forma mais favorável do que aqueles que não receberam nenhum material (Figura 1 ). Isto pode ter ocorrido em função dos materiais informativos, mesmo sendo simples como um folheto ou folder, aumentarem o índice de retenção da informação, facilitando $o$ aprendizado ${ }^{16}$.

Pelo fato de que poderiam participar da pesquisa pais e cuidadores atendidos nos serviços de audiologia fornecidos no Campus onde o "Portal dos Bebês" foi desenvolvido, optou-se pela avaliação em formato anônimo e sem a exigência de cadastro do participante, para que este se sentisse a vontade para expressar sua real opinião a respeito do website. Deste modo, não foi a proposta do presente estudo realizar avaliação do conhecimento dos pais a respeito do AASI e molde auricular "pré" e "pós" navegação no website, já que sem o cadastro não seria possível comparar as respostas de um mesmo participante, em dois momentos distintos.

Assim, os pais/cuidadores foram solicitados a navegar pela seção "Aparelhos Auditivos" e realizar uma autocrítica a respeito do seu conhecimento sobre estes conteúdos, previamente ao acesso ao webiste. Os participantes foram depois questionados sobre o quanto o "Portal dos Bebes" auxiliou no entendimento de determinado conteúdo, em comparação ao seu conhecimento prévio. Destaca-se que existe subjetividade nesta análise, sendo esta uma limitação do estudo.

Observou-se que grande parte dos pais e cuidadores $(n=10,45,5 \%)$ julgaram seu conhecimento prévio a respeito dos AASIs como sendo bom ou muito bom. Isto pode ter ocorrido em função da experiência que os mesmos já possuíam com uso dos AASIs em seus filhos, visto que, em média, o tempo de adaptação do AASI foi de 26,5 meses (Tabela 1).

Quando considerados todos os participantes que avaliaram pelo menos um item do questionário de conteúdo (Tabela 5) observou-se que as pontuações variaram de 3,1 ("verificação do AASI / resolução de problemas") a 3,5 ("tecnologia dos AASIs" e "tipos de AASIs"). Este resultado é extremamente favorável considerando que a pontuação máxima (correspondente a "ajudou muito") para cada item era igual a quatro. Uma alta pontuação total também foi obtida.

Não foram encontradas diferenças estatisticamente significantes entre os conteúdos avaliados. No entanto, verificou-se que o item "verificação do AASI / resolução de problemas" recebeu a menor pontuação. Isto pode ter corrido em virtude deste conteúdo consistir apenas por texto e Tabela explicativa. A combinação de áudio, texto e vídeo cria uma mistura de múltiplos sentidos permitindo melhor apreensão do conteúdo ${ }^{17}$. Desta forma é sugerida a reformulação deste conteúdo, de forma a privilegiar mais o uso de animações e vídeos demonstrativos.

A maior pontuação atribuída para o item "como criar uma rotina de uso do AASI" provavelmente ocorreu em função deste item abordar aspectos que foram relacionados como principais dificuldades dos pais a respeito do uso do AASI pela criança. Quando considerados juntos os aspectos de fixação do AASI, tempo de uso diário do AASI e habituação da criança com o uso do AASI (Tabela 6) corresponderam a $50 \%$ das dificuldades elencadas pelos participantes.

A correlação negativa entre o conhecimento prévio a respeito dos tópicos em AASI e a pontuação 
total dada para o conteúdo do website era esperada, tendo em vista que as perguntas formuladas para avaliação do conteúdo diziam respeito ao quanto o website auxiliou no entendimento de uma informação quando comparada ao conhecimento prévio. Desta forma, se o participante já possuía um conhecimento prévio bom ou muito bom sobre o assunto a tendência era de que o website forneceria menor auxílio.

Conforme dito anteriormente as principais dificuldades $(50 \%)$ elencadas pelos pais a respeito do AASI na criança (Tabela 6) diziam respeito ao estabelecimento de uma rotina de uso. Em seguida o uso e cuidados com o aparelho de amplificação sonora individual $(14,3 \%)$ e a limitações que o AASI impõe para a realização de algumas atividades $(14,3 \%)$ como tomar banho, brincar na areia e ir à piscina foram citados. Observou-se que o website ajudou ou ajudou muito doze participantes $(63,2 \%)$ na resolução destas dificuldades elencadas. É necessário atualizar o conteúdo do "Portal dos Bebês" com informações sobre as estratégias de comunicação que podem ser utilizadas nos momentos em que a criança não esteja utilizando o AASI (por exemplo, hora do banho).

Finalmente, verificou-se que aproximadamente $76 \%$ dos pais estão satisfeitos ou muito satisfeitos com o website. No entanto, deve ser destacado que quatro participantes (19\%) estavam insatisfeitos ou muito insatisfeitos com o mesmo. Estes participantes não realizaram comentários a respeito dos motivos que levaram à insatisfação. Ainda assim todos os participantes relataram que indicariam o website para outros pais de crianças deficientes auditivas.
No que se refere aos comentários dos participantes (Figura 3) observou-se que estes solicitam a inclusão de conteúdos sobre aspectos de terapia e formas para lidar com a perda de audição no caso de crianças mais velhas. Este comentário pode ser justificado pela idade atual da criança (média de 53 meses) sobre a qual os respondentes se referiram durante o preenchimento do questionário.

\section{CONCLUSÃO}

A grande maioria dos pais participantes nesta pesquisa considerou seu conhecimento sobre o AASI como sendo bom ou muito bom, em função de já terem experiência com o uso dos mesmos em seus filhos. Esta experiência influenciou as pontuações fornecidas para o website bem como a satisfação com o mesmo. No entanto, os pais atribuíram pontuações bastante favoráveis para os conteúdos apresentados e $76,2 \%$ destes estavam satisfeitos ou muito satisfeitos com o "Portal dos Bebês".

O website se mostrou útil na complementação da orientação fornecida aos pais de crianças deficientes auditivas usuárias de aparelhos de amplificação sonora individuais. Outros estudos com um maior número de pais participantes são necessários. Os resultados preliminares indicam que o "Portal dos Bebês" é um instrumento que pode auxiliar pais de crianças deficientes auditivas na compreensão das orientações fornecidas no momento da adaptação do AASI. A continuidade do estudo com maior número de participantes é necessária.

\section{ABSTRACT}

Purpose: to verify the self-assessment of knowledge of parents of hearing impaired children regarding hearing aids (HA) and earmolds and how the content of the "Babies'Portal - Hearing Section" website supported the understanding of such information. Methods: 22 parents (six men and 16 women, mean age 32,6 years) completed an anonymous online form available on the website with 36 questions about demographics, internet use and the guidance provided the time of HA fitting. Also answered questions about the quality of website content. Results: on the fitting of hearing aids informational materials were provided only for $52,4 \%$ of parents, however, they were satisfied with the orientation. On average the helped provided by the website content was $86 \%$. The score for satisfaction with the content and the website was related to the child's age, duration of use of hearing aids and prior knowledge of parents about the topics on the hearing aid and ear mold. Conclusion: preliminary results indicate that the "Babies' Portal" is an instrument that can help parents of hearing impaired children in the understanding of the guidance given at the time of HA fitting. The continuity of the study with a larger number of participants is required.

KEYWORDS: Hearing Loss; Hearing Aids; Audiology; Counseling; Telemedicine; Internet 


\section{REFERÊNCIAS}

1. Elfeibein JL, Bentler RA, Davis JM, Niebuhr DP. Status of school children's hearing aids relative to monitoring practices. Ear Hear. 1988;9(4):212-7.

2. Robbins AM. Empowering parents to help their newly diagnosed child gain communication skills. Hear J. 2002;55(11):55-9.

3. Jackson CW, Wegner JR, Turnbull AP. Family quality of life following early identification of deafness. Lang Speech Hear Serv Sch. 2010;41(2):194-205.

4. Fitzpatrick E, Graham ID, Durieux-Smith A, Angus $D$, Coyle D. Parents' perspectives on the impact of the early diagnosis of childhood hearing loss. Int $\mathrm{J}$ Audiol. 2007;46(2):97-106.

5. Fitzpatrick E, Angus D, Durieux-Smith A, Graham ID, Coyle D. Parents' needs following identification of childhood hearing loss. Am J Audiol. 2008;17(1):38-49.

6. Strömberg A. The crucial role of patient education in heart failure. Eur J Heart Fail. 2005;7(3):363-9.

7. Ministério da Saúde. Portaria SAS 587 de 07 de outubro de 2004, Anexo IV. Diretrizes para o fornecimento de aparelhos de amplificação sonora individual (AASI). Disponível em: http:// dtr2001.saude.gov.br/sas/PORTARIAS/Port2004/ PT-587\%20Anexo\%20IV.htm.

8. Ministério da Saúde - Sistema de Informações Ambulatoriais do SUS (SIA/SUS). Acesso em: jan 2010. Disponível em: http://tabnet.datasus.gov.br/ cgi/tabcgi.exe?sia/cnv/pauf.def.

9. Vieira HC, Castro AE, Júnior Schuch VF. O uso de questionários via e-mail em pesquisas acadêmicas sob a ótica dos respondentes. XIII SEMEAD Seminários em administração. 2010. Disponível em: http://www.ead.fea.usp.br/semead/13semead/ resultado/trabalhosPDF/612.pdf.

10. Fan W, Yan Z. Factors affecting response rates of the web survey: A systematic review. Comput Human Behav. 2010;26(2):132-9.

11. Mikkelsen UJ, Nielsen P, Rasmussen S. Support services in Denmark for parents of children who are deaf or hard of hearing - a national survey. Scand Audiol Suppl. 2001;53:116-9.

12. Roush J, Harrison M. Age of suspicion, identification and intervention for young children with hearing loss. In: Bess FH. Children with hearing impairment: contemporary trends. Nashville: Vanderbilt Bill Wilkerson Center Press, 1998. p. 25-32.

13. Ingber S, Dromi E. Actual versus desired family - centered practice in early intervention for children with hearing loss. J Deaf Stud Deaf Educ. 2010;15(1):59-71.

14. Kessels RPC. Patients' memory for medical information. J R Soc Med. 2003;96(5):219-22.

15. Ferrari DV, Tomé $T$, Bastos BG. Internet based hearing aid orientation. British Academy of Audiology Convention, Liverpool, 2008.

16. Wilson EAH, Park DC, Curtis LM, Cameron KA, Clayman ML, Makoul G et al. Media and memory: The efficacy of video and print materials for promoting patient education about asthma. Patient Educ Couns. 2010;80(3):393-8.

17. Murphy KR. Computer-based patient education. Otolaryngol Clin North Am. 1998;31(2):309-17.

http://dx.doi.org/10.1590/1982-0216201413412

Recebido em: 02/06/2012

Aceito em: 21/10/2012

Endereço correspondência:

Bárbara Guimarães Bastos

Rua M.M.D.C, no 539, apto 143 - Butantã

São Paulo - SP

CEP: 05510-021

E-mail: barbarabastos@gmail.com 